Journal of Computer Science 8 (5): 789-795, 2012

ISSN 1549-3636

(C) 2012 Science Publications

\title{
Performance Analysis of Multi Spectral Band Image Compression using Discrete Wavelet Transform
}

\author{
Kousalyadevi, R. and S.S. Ramakrishnan \\ Institute of Remote Sensing, \\ College of Engineering, Guindy, Anna University, Chennai, India
}

\begin{abstract}
Problem statement: Efficient and effective utilization of transmission bandwidth and storage capacity have been a core area of research for remote sensing images. Hence image compression is required for multi-band satellite imagery. In addition, image quality is also an important factor after compression and reconstruction. Approach: In this investigation, the discrete wavelet transform is used to compress the Landsat5 agriculture and forestry image using various wavelets and the spectral signature graph is drawn. Results: The compressed image performance is analyzed using Compression Ratio (CR), Peak Signal to Noise Ratio (PSNR). The compressed image using dmey wavelet is selected based on its Digital Number Minimum (DNmin) and Digital Number Maximum (DNmax). Then it is classified using maximum likelihood classification and the accuracy is determined using error matrix, kappa statistics and over all accuracy. Conclusion: Hence the proposed compression technique is well suited to compress the agriculture and forestry multi-band image.
\end{abstract}

Key words: Compression Ratio (CR), Digital Number minimum (DNmin), Peak Signal to Noise Ratio (PSNR), Remote Sensing (RS), Mean Square Error (MSE)

\section{INTRODUCTION}

Image compression on digital images reduces the redundancy in storing or transmitting the information in an efficient form (Al-Sammraie, 2001). It explores three types of redundancies: coding redundancy, inter pixel redundancy and psycho visual redundancy (Dwivedi et al., 2004). Image compression can be loss less and lossy. In lossless compression, there is a perfect reconstruction of original data after decompression but the storage capacity required and transmission bandwidth are high (Gupta and Mutha, 2003).

The Lossy compression reduces the size of the data needed to store it along with a considerable loss of information. Typically in a digital true-colour image, each colour component is quantized to 8 bits and hence a colour is specified with 224 bits. Moreover, a colour image contains lot of data redundancy and requires a large amount of storage space. Image compression reduces the number of possible colours of bits required to represent it, by removing spatial and spectral redundancies. This reduces transmission and storage cost. Major parameters to be considered in lossy compression scheme are CR, PSNR, DNmin and DNmax.

The compression technique DCT is closer to DFT, used in JPEG for image compression technique. In JPEG quantization matrices are used for quantizing the image and different quantization matrix is to be specified for each colour component. DCT is used for block processing which leads to blocking artifacts. JPEG-2000 standard employs wavelet for compression due to its merits in terms of scalability, localization and energy concentration. It supports decomposition of all the sub-bands at each level and hence requires full decomposition at a certain level. But it suffers from ringing and blurring artifacts. For colour image compression techniques based on moment-preserving and block truncation coding; input image is divided into non overlapping blocks and each block is assigned to a colour. A bit map is then generated for each block to represent the pixel colours. But only compression ratio up to about 13 is achieved.

In continuous wavelet transform the entire signal is multiplied by scaled and shifted versions of the wavelet. This process produces wavelet coefficients that are functions of scale and position. It provides better image quality especially when high compression is required. But it has limited resolution especially in low frequency region. Several studies attempted to compress the image using Haar wavelet and the quality is measured using CR and PSNR (Kanvel and Monie, 2009). The parameters such as CR and PSNR (Sadashivappa and Babu, 2008) are used to estimate the quality of the image. The CR is employed to compare the

Corresponding Author: Kousalyadevi, R., Institute of Remote Sensing, Research Scholar, College of Engineering, Guindy, Anna University, Chennai, India 
compressed images using various wavelets and PSNR to calculate highest signal to noise power. These parameters are applicable for three band general color images.

But for Remote Sensing (RS) images, image signature plays an important role in classifying the data. Hence in this study, the image signatures are compared using spectral signature graph. RS data generally collected from the sensors on satellite platform are used for mapping land features. While using imagery mode, spectral signature curve is used extensively to understand and classify the feature of the land. Three band compressed color images are useful for visual quality but the RS data is used for feature extraction and classification of earth features using mathematical techniques. The digital numbers are rigorously processed using statistical technique. In this study, DWT (Buccigrossi and Simoncelli, 1999) is used for compressing the multi spectral Landsat5 image. Wavelets are advantageous over Fourier methods in analyzing the discontinuities and sharp spikes in image. Wavelet Transform (WT) is used to reduce the inter pixel redundancy in an image and the performance is analyzed using CR, MSE and PSNR. Based on this, some of the wavelets are selected and its DN values are calculated. Using these DN values the spectral signature graph is drawn. From the graph, a particular wavelet is selected based on the DN values. After image compression, the image classification is used to identify the liability between the original and compressed image. In this study, Maximum Likelihood classification is used to identify the information classes of interest and a statistical characterization of the reflectance for each class is developed and accuracy is assessed.

\section{MATERIALS AND METHODS}

Satellite imagery: Landsat5 multi-spectral images of band 1 to 5 and 7 raw data are downloaded from the GLCF website [GLCF, 2010]. Subset images were obtained from the raw data using ERDAS 8.5 software. These subset images are compressed using MATLAB7.8.0 and accuracy assessment is made by classifying the original image and compressed image using ERDAS 8.5.

Original landsat5 image: The subset of the satellite image of size $342 \times 342 \times 6$ is taken from the raw image of size $8106 \times 7064 \times 6$ and shown in Fig. 1. An agriculture and forest image of Stavropol'skiy Kray, Russia is taken. The spectral bands $1-5$ and 7 are downloaded. Band 6 is not considered because of $120 \mathrm{~m}$ resolution Image compression using DWT: The DWT is fast and its linear operation operates on a data vector whose length is an integer power of two, transforming it into numerically different vectors of same length. It has high decorrelation (Grgic et al., 2001) and energy compaction efficiency. In DWT, the decomposition of the image is produced by analysis filter bank followed by down sampling. Reconstruction is done by upsampling followed by synthesis filter bank. The decomposition (Agrawal and Venugopalan, 2009) changes the proportion of detail coefficients in the image. The detailed coefficients are reduced by fixing a threshold value to obtain higher compression rates (Mallat, 1989). The best trade-off (Heung-Yeung et al., 2003) between energy loss and compression is provided by decomposing at level 3 for heterogeneous images.

Evaluation of image quality: The subset image is decomposed at decomposition level 3. After fixing a level dependent threshold, the image is compressed using various wavelets such as Haar, Daubechies, Coiflet, Symlet and Discrete Meyer. Parameters like MSE and PSNR are calculated. If the PSNR is high then the $\mathrm{CR}$ and $\mathrm{DN}$ are calculated. Using the DN values spectral signature graph is drawn. The standard measures of determining the image quality are Mean Square Error (MSE) and Peak Signal to Noise Ratio (PSNR). MSE is defined as the ratio of square of the difference between original image and compressed image to the total number of pixels in the original image. PSNR is defined as the ratio of the total number of pixels in the compressed image to the mean square error in $\mathrm{dB}$. Let $\mathrm{I} 1(\mathrm{~m}, \mathrm{l})$ be the original image, $\mathrm{I} 2(\mathrm{~m}, \mathrm{~L})$ be the compressed image and $\mathrm{M}$ and $\mathrm{L}$, the row and column of the original image then MSE is given in Eq.1:

$\mathrm{MSE}=\frac{\left.\sum_{\mathrm{M}<\mathrm{L}}\left[\mathrm{I}_{1},(\mathrm{~m}, \mathrm{I})\right]-\mathrm{I}_{2}(\mathrm{~m}, \mathrm{I})\right]^{2}}{\mathrm{M}^{*} \mathrm{~L}}$

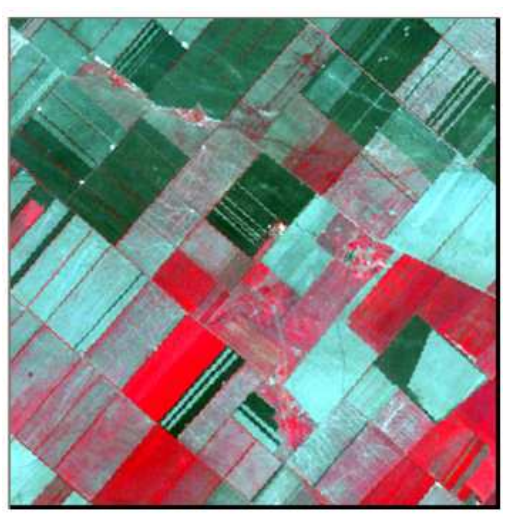

Fig. 1: Original image $\mathrm{f}$ size $342 \times 342 \times 6$ 


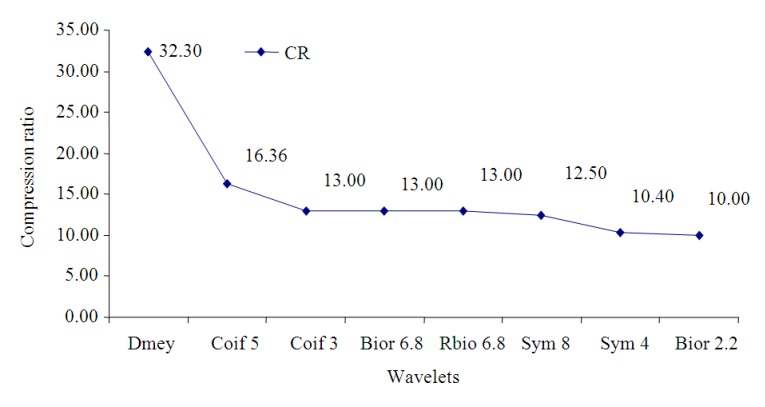

Fig. 2: Compression ratio

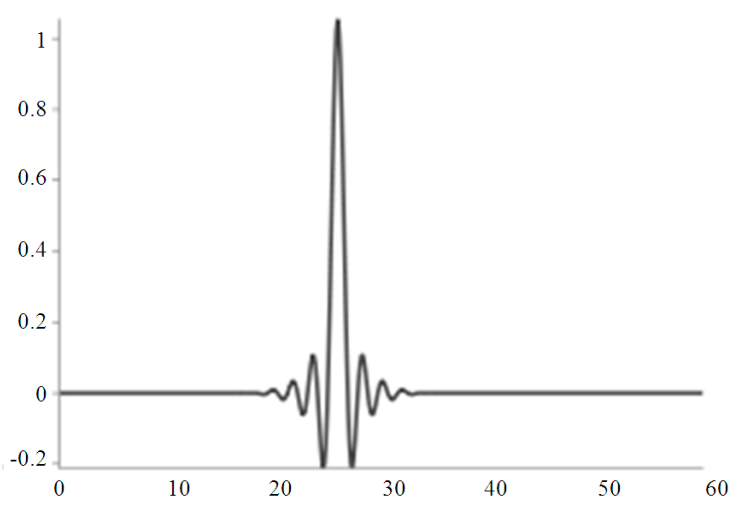

Fig. 3: Dmey scaling function $\varphi$

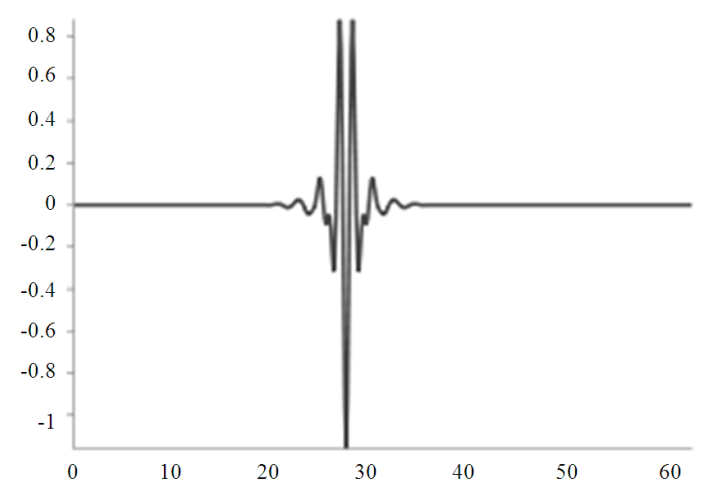

Fig. 4: Wavelet function $\psi$

The peak signal to noise ratio is given in Eq. 2:

$\mathrm{PSNR}=10 \log 10$ [342*343/ MSE $]$

The image quality is measured by using DNminimum and DNmaximum. Using Eq. 1 and 2, the image quality is analyzed for various wavelets. When the PSNR of the compressed image is high, CR and DN values of those images are calculated. If the PSNR value is low, then that particular wavelet is neglected.
Based on this, a number of wavelets are selected, as in Table 1 and the CR is calculated. The wavelets are then selected based on high CR and is shown in Fig. 2. After calculating the $\mathrm{CR}$, the $\mathrm{DN}$ values of those compressed images are calculated and are given in Table 2. It presents minimum and maximum pixel values of the compressed image for selected wavelets.

Discrete Meyer wavelet: This wavelet is a FIR based approximation of the Meyer wavelet. The properties of dmey are symmetric, orthogonal and biorthogonal. The Meyer wavelet and scaling function are defined in the frequency domain and shown in Fig. 3 and 4. The wavelet functions are given in Eq. 3 and scaling functions in Eq. 4:

$$
\begin{aligned}
& \hat{\psi}(\omega)=(2 \pi)^{-\frac{1}{2}} \mathrm{e} \frac{\mathrm{i} \omega}{2} \sin \left(\frac{\pi}{2} \mathrm{v}\left(\frac{3}{2 \pi}|\omega|-1\right)\right) \text { if } \frac{2 \pi}{3}<|\omega|<\frac{4 \pi}{3} \\
& \hat{\psi}(\omega)=(2 \pi)^{\frac{1}{2}} \mathrm{e} \frac{\mathrm{i} \omega}{2} \cos \left(\frac { \pi } { 2 } \mathrm { v } \left(\frac{\pi}{2} \mathrm{v}\left(\frac{3}{4 \pi}|\omega|-1\right) \text { if } \frac{4 \pi}{3}<|\omega|<\frac{8 \pi}{3}\right.\right. \\
& \hat{\psi}(\omega)=0 \mathrm{if}|\omega| \notin\left[\frac{2 \pi}{3}, \frac{8 \pi}{3}\right]
\end{aligned}
$$

Where:

$$
v(a)=a^{4}\left(35-84 a+70 a^{2}-20 a^{3}\right) a \in[0,1]
$$

And:

$$
\begin{aligned}
& \hat{\phi}(\omega)=(2 \pi)^{-1 / 2} \text { if }|\omega| \leq \frac{2 \pi}{3} \\
& \left.\hat{\phi}(\omega)=(2 \pi)^{-1 / 2} \cos \left(\frac{\pi}{2} \mathrm{v}\left(\frac{\pi}{2}|\omega|-1\right)\right) \text { if } \frac{2 \pi}{3}|\omega| \leq \frac{4 \pi}{3}\right) \\
& \hat{\phi}(\omega)=0 \text { if }|\omega|>\frac{4 \pi}{3}
\end{aligned}
$$

Spectral signature graph: Spectral signatures are plots that compare the percentage reflectance values of landscape features such as road, forest, water and sand. Differences among spectral signatures are used to classify the remotely sensed images into classes of landscape features. Using the DNmin and DNmax values given in Table 2, the spectral signature graph is drawn and shown in Fig. 5. The wavelets Sym4, Sym8, Coif 3, Coif 5, Bior 2.2, Bior 6.8, Rbio 6.8 and dmey are shown in the graph based on the DN values. These wavelets are closer to the DN values of the original image. Dmey wavelet alone is selected for compressing the Landsat 5 agriculture and forest image, as it provides high CR and PSNR. The DN values of the compressed image approximately equal the original image compared to any other wavelets. 


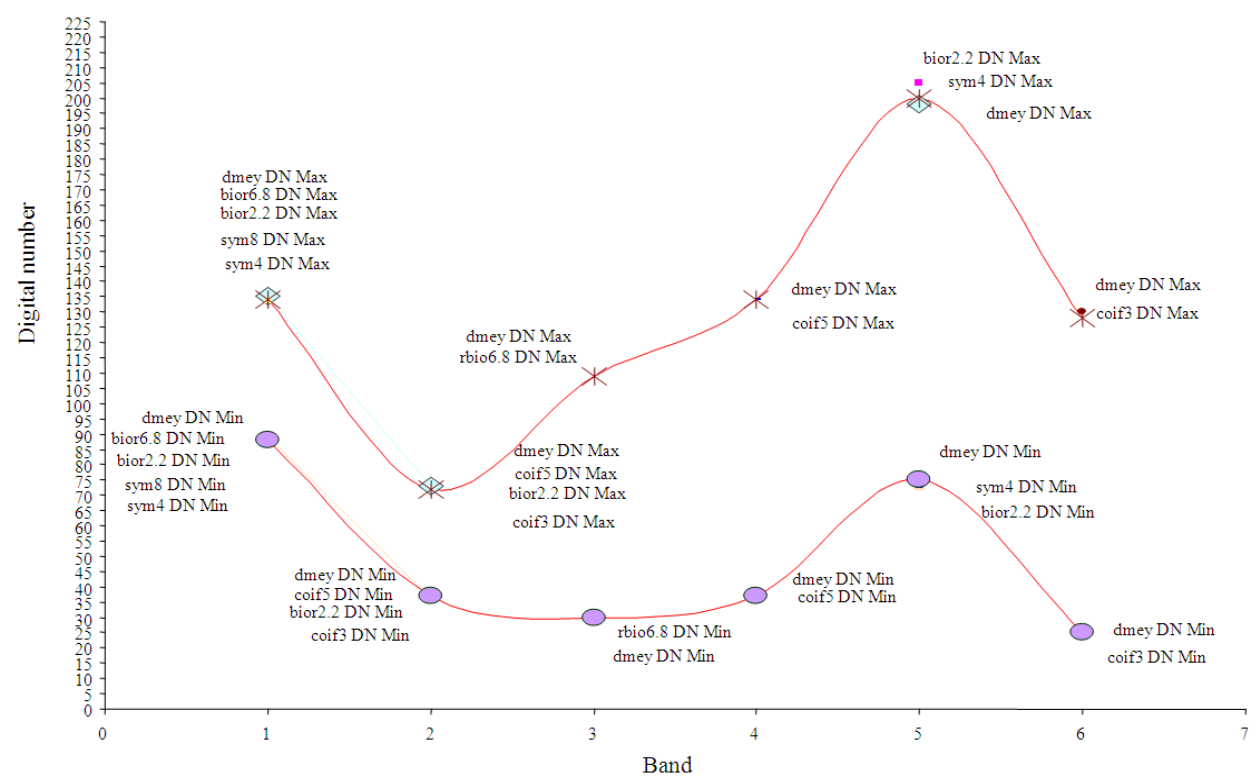

Fig. 5: Spectral signature for the wavelet functions

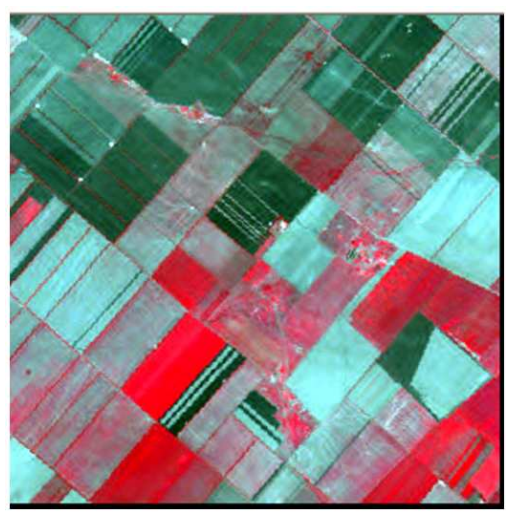

Fig. 6: Compressed image using Dmey wavelet at decomposition level 3

The compressed image using dmey wavelet is shown in Fig. 6. This spectral signature graph proves that the dmey compressed image is same as the original image.

Image classification: RS image classification plays the very important practical vital role in geological survey and mineral exploration. The neural network technology is an important for the RS image classification. But, the BP NN still has short comings, for example, the learning coverage is slow and he training process is easy to fall into the partial minimum. Image classification uses the spectral information represented by the digital numbers in one or more spectral bands and classifies each individual pixel based on this spectral information (Lillesand et al., 2008).
Table 1: PSNR for few wavelet functions for every band

\begin{tabular}{lllllll}
\hline Wavelets & Band 1 & Band 2 & Band 3 & Band 4 & Band 5 & Band 7 \\
\hline Dmey & 45.54 & 49.29 & 44.780 & 43.93 & 39.49 & 44.01 \\
Haar & 42.50 & 45.84 & 40.650 & 39.24 & 34.85 & 36.23 \\
Coif1 & 43.36 & 46.87 & 41.900 & 40.62 & 36.20 & 37.69 \\
Coif 3 & 44.03 & 47.70 & 42.920 & 41.77 & 37.37 & 38.84 \\
Coif 5 & 44.38 & 48.10 & 43.380 & 42.27 & 37.89 & 39.35 \\
Db 7 & 43.83 & 47.38 & 42.550 & 41.33 & 36.99 & 38.41 \\
Db 9 & 43.89 & 47.47 & 42.599 & 41.38 & 37.09 & 38.48 \\
Sym 4 & 43.67 & 47.28 & 42.410 & 41.17 & 36.84 & 38.27 \\
Sym 8 & 43.99 & 47.65 & 42.850 & 41.71 & 37.32 & 38.74 \\
Bior 2.2 & 43.45 & 47.06 & 42.170 & 40.94 & 36.61 & 38.11 \\
Bior 6.8 & 44.08 & 47.78 & 43.000 & 41.89 & 37.55 & 38.96 \\
Rbio 6.8 & 43.87 & 47.52 & 42.670 & 41.47 & 37.13 & 38.54 \\
Rbio 3.3 & 39.73 & 42.68 & 37.120 & 35.58 & 30.93 & 32.24 \\
\hline
\end{tabular}

Table 2: DN values for the chosen wavelet functions

\begin{tabular}{|c|c|c|c|c|c|c|c|}
\hline Wavelets & Pixel value & Band 1 & Band 2 & Band 3 & Band 4 & Band 5 & Band 7 \\
\hline \multirow[t]{2}{*}{$\overline{\text { Sym } 4}$} & DN Min & 89 & 35 & 27 & 33 & 73 & 16.0 \\
\hline & DN Max & 135 & 70 & 106 & 133 & 205 & 131.0 \\
\hline \multirow[t]{2}{*}{ Sym 8} & DN Min & 88 & 36 & 28 & 36 & 74 & 23.0 \\
\hline & DN Max & 134 & 70 & 107 & 134 & 202 & 130.0 \\
\hline \multirow[t]{2}{*}{ Coif 3} & DN Min & 87 & 36 & 28 & 36 & 75 & 24.0 \\
\hline & DN Max & 133 & 72 & 110 & 133 & 200 & 130.0 \\
\hline \multirow[t]{2}{*}{ Coif 5} & DN Min & 87 & 36 & 27 & 37 & 75 & 20.0 \\
\hline & DN Max & 134 & 72 & 109 & 134 & 203 & 131.0 \\
\hline \multirow[t]{2}{*}{ Bior 2.2} & DN Min & 88 & 36 & 28 & 35 & 72 & 20.0 \\
\hline & DN Max & 135 & 73 & 108 & 131 & 198 & 130.0 \\
\hline \multirow[t]{2}{*}{ Bior 6.8} & DN Min & 89 & 35 & 29 & 36 & 75 & 22.0 \\
\hline & DN Max & 134 & 71 & 107 & 131 & 197 & 130.0 \\
\hline \multirow[t]{2}{*}{ Rbio 6.8} & DN Min & 88 & 34 & 29 & 34 & 76 & 24.0 \\
\hline & DN Max & 133 & 72 & 110 & 135 & 199 & 131.0 \\
\hline \multirow[t]{2}{*}{ Dmey } & DN Min & 88 & 37 & 30 & 37 & 75 & 25.0 \\
\hline & DN Max & 134 & 72 & 109 & 134 & 200 & 128.0 \\
\hline \multirow[t]{2}{*}{ Original } & DN Min & 89 & 37 & 32 & 39 & 71 & 25.0 \\
\hline & DN Max & 135 & 73 & 111 & 128 & 201 & 128.0 \\
\hline
\end{tabular}

The original image and compressed image are compared to find the accuracy through image classification (Jain, 1989). RS image classification 
plays a vital role in the geological survey and mineral exploration. The two types of image classification are supervised classification and unsupervised classification. In supervised classification, spectral signatures are developed from specified locations in the image. These specified locations are specified as 'training sites' and are defined by the expert. Once the training sites are developed, this information can be used along with various images of different bandwidths, to create spectral signatures from the specified areas. These signatures are used to classify all pixels in the scene.

Maximum likelihood classification: Maximum likelihood is one of the supervised classification which uses the Gaussian threshold stored in each class signature to determine if a given pixel falls within the class or not (Jain, 1989). The threshold is the radius of a hyper ellipse surrounding the mean of the class in feature space. The pixel falling inside the hyper ellipse is assigned to the class, else assigned to the null class. The maximum likelihood classifier gives more accurate results than parallelepiped classification.

In this study, supervised Maximum likelihood Classification is used for accuracy assessment. It is used to obtain the training data called as signatures and these are used to generate class signatures (area of interest called AOI-vegetation, forestry). These signatures are parametric as they have statistical information. Using ERDAS 8.5, each signature is labeled and a colour is assigned. Applying this discriminant function to the entire feature space, all pixels in the original image are labeled. Thus the output map consists of one label for each AOI. Then region growing concept is applied using seed properties to grow the pixels in other areas.

Only those pixels above, below, to the left and to the right of the seed are considered contiguous. Thematic raster layer and distance image files are created to display the output. The end result is numerical image data converted into descriptive labels that categorize different forest and agriculture regions. Hence the multi-spectral image consisting of several bands has been reduced to a map consisting of category labels. The same training data is used to classify the Dmey compressed image.

Measurement of image accuracy: The random points are then generated to perform the accuracy assessment. These points are selected from the Dmey compressed image and are compared with the reference data from the original image. Using these values the accuracy assessment is done. The accuracy assessment consists of error matrix, accuracy totals and kappa statistics.
After evaluation of selected sample points in each reference data set, an error matrix is constructed. Comparing classified data labels to reference data labels for each classification, overall accuracy, class specific user and producer accuracy are calculated. The users accuracy specifies the number of correct classifications as given in Eq. 6. The producers' accuracy is a measure of how much of the land in each category is classified correctly. The overall accuracy is defined as the ratio of the proportion of the total number of correctly classified pixels to the total number of pixels in the matrix given in Eq. 5 and 6 is expressed in percentage:

An overall measure of accuracy

$=\frac{\text { Total number of correct classifications }}{\text { Total Number of classifications }}$

Users accuracy

$$
=\frac{\text { Number of correct classifications }}{\text { Total number of classifications in the category }}
$$

Another measure of map accuracy is the kappa coefficient, which is a measure of the proportional improvement by the classifier over a purely random assignment to classes. The kappa value indicates the accuracy of output classification. Comparison is made between originally classified image and classified Dmey compressed image to find the accuracy.

\section{RESULTS}

All the wavelets such as Haar, Symlet, Coiflet, Daubechies, Biorthogonal Discrete Meyer and reverse Biorthogonal wavelets are applied over the original subset image and MSE and PSNR values of all the compressed images are calculated. The compressed images using the wavelets Dmey, Haar, Coif1, Coif3, Coif5, Db7, Db9, Sym4, Sym8, Bior2.2, Bior6.8, Rbio6.8 and Rbio3.3 are selected based on high PSNR and is shown in Table 1.

Table 3: Error matrix

\begin{tabular}{|c|c|c|c|c|c|c|c|}
\hline \multirow{2}{*}{$\begin{array}{l}\text { Classified } \\
\text { Data }\end{array}$} & \multicolumn{4}{|c|}{ Unclassified data } & \multicolumn{3}{|c|}{ Reference data } \\
\hline & & Agril & Agri2 & Agri3 & Forest1 & Forest2 & Row total \\
\hline Unclassified & 0 & 0 & 0 & 0 & 0 & 0 & 0 \\
\hline Agril & 0 & 6 & 0 & 0 & 0 & 0 & 6 \\
\hline Agri 2 & 0 & 0 & 2 & 0 & 0 & 0 & 2 \\
\hline Agri 3 & 0 & 0 & 0 & 5 & 0 & 0 & 5 \\
\hline Forest 1 & 0 & 0 & 0 & 0 & 1 & 0 & 1 \\
\hline Forest 2 & 0 & 0 & 0 & 1 & 0 & 5 & 6 \\
\hline Column total & 0 & 6 & 2 & 6 & 1 & 5 & 20 \\
\hline
\end{tabular}


Table 4: Accuracy totals

\begin{tabular}{llllcc}
\hline Class name & \multicolumn{2}{l}{$\begin{array}{l}\text { Reference Classified } \\
\text { Totals }\end{array}$} & $\begin{array}{l}\text { Number } \\
\text { totals }\end{array}$ & $\begin{array}{l}\text { Producers } \\
\text { correct }\end{array}$ & $\begin{array}{l}\text { Users } \\
\text { accuracy }(\%)\end{array}$ \\
accuracy (\%)
\end{tabular}

Table 5: Conditional kappa for each category

\begin{tabular}{ll}
\hline Class name & Kappa \\
\hline Unclassified & 0.0000 \\
Agri 1 & 1.0000 \\
Agri 2 & 1.0000 \\
Agri 3 & 1.0000 \\
Forest 1 & 1.0000 \\
Forest 2 & 0.7778 \\
\hline
\end{tabular}

After calculating the PSNR, the CR of these images is calculated. Some of the compressed images using wavelets Dmey, Coif5, Coif3, Bior6.8, Rbio6.8, Sym8, Sym4, Bior2.2 are selected based on high CR and is shown in Fig. 2. The final selection of compressed image is done by calculating the $\mathrm{DN}$ values of the compressed image.

Considering the DN values, Sym4 provides the exact $\mathrm{DN}$ values $(\mathrm{DNmin}=89, \mathrm{DNmax}=135)$ for band1, Dmey provides the DN values $(\mathrm{DNmin}=37$, DNmax $=72$ ) for band2, Dmey provides the DN values $(\mathrm{DNmin}=30, \mathrm{DNmax}=109)$ for band3, Bior6.8 provides the DN values $(\mathrm{DNmin}=36, \mathrm{DNmax}=131)$ for band4, Dmey provides DN values $(\mathrm{DNmin}=75, \mathrm{DNmax}=200)$ for band6 and Dmey provides DN values $(\mathrm{DNmin}=25$, DNmax $=128$ ) for band7 Table 2. Using these values the spectral signature graph is drawn.

The accuracy assessment report includes error matrix, as in Table 3, accuracy total, as in Table 4 and Kappa statistics as in Table 5. It is found that overall classification accuracy is at $95.00 \%$ and overall Kappa Statistics is at 0.9331 .

\section{DISCUSSION}

In this study, the compression of multi spectral band Landsat5 image using DWT is discussed. Compared to all the wavelet functions, Dmey wavelet provides high PSNR for band1 (45.54), band2 (49.29), band3 (44.78), band4 (43.93), band5 (39.49) and band7 (44.01) at decomposition level 3. While considering the CR, Dmey wavelet provides the $32.3 \%$ compression compared to other wavelet function and Dmey provides the DN values which is approximately equal to the original image compared to all the wavelet functions.
The accuracy assessment is done by classifying the original image and the Dmey compressed image. Random reference points are selected from the original forest and agriculture image and these points are used to classify the Dmey compressed image. The error matrix in Table 4 shows an error in forest 2 . This leads to an overall classification accuracy of $95.00 \%$ and the overall Kappa statistics of 0.9331 as shown in Table 5.

\section{CONCLUSION}

In this study, use of 2D Discrete Wavelet Transform for compression of Landsat5 agriculture and forestry satellite image has been discussed and its performance analyzed by CR, PSNR, DNmin and DNmax. All wavelet functions are compared using spectral signature graph. Finally it has been proved that, Discrete Meyer Wavelet (Dmey) provides the compressed image with high PSNR for each band, relatively high compression ratio (32.3) and pixel value equal to the original image. Then the compressed image is classified using maximum likelihood classification. Error matrix, over all accuracy matrix and kappa statistics are applied over the dmey classified image for accuracy assessment. It is also evident that Dmey compressed image provides better classification accuracy compared with other wavelets.

\section{REFERENCES}

Agrawal, N. and K. Venugopalan, 2009. SAR image compression using wavelet packets. Proceedings of the International Conference on Signal Processing Systems, May 15-17, IEEE Explore Press, Singapore, pp: 343-347. DOI: 10.1109/ICSPS.2009.54

Al-Sammraie, M.F.H., 2011. Colored image compression using gradient adjustment prediction based wavelet. J. Comput. Sci., 7: 304-313. DOI: 10.3844/jcssp.2011.304.313

Buccigrossi, R.W. and E.P. Simoncelli, 1999. Image compression via joint statistical characterization in the wavelet domain. IEEE Trans. Image Process., 8: 1688-1701. DOI: 10.1109/83.806616

Dwivedi, R.S., S. Kandrika and K.V. Ramana, 2004. Comparison of classifiers of remote-sensing data for land-use/land-cover mapping. Curr. Sci., 86: 328-334.

Grgic, S., M. Grgic and B. Zovko-Cihlar, 2001. Performance analysis of image compression using wavelets. IEEE Trans. Indus. Elect., 48: 682-695. DOI: $10.1109 / 41.925596$ 
Gupta, D. and S. Mutha, 2003. Image compression using wavelet packets. Proceedings of the Conference on Convergent Technologies for AsiaPasific Region, Oct. 15-17, IEEE Explore Press, India, $\quad$ pp: $922-926 . \quad$ DOI: 10.1109/TENCON.2003.1273382

Heung-Yeung, S., S.B. Kang and S.C. Chan, 2003. Survey of image-based representations and compression techniques. IEEE Trans. Circ. Syst. Video Technol., 13: 1020-1037. DOI: 10.1109/TCSVT.2003.817360

Jain, A.K., 1989. Fundamentals of Digital Image Processing. 5th Edn., Prentice Hall, Englewood, ISBN: 0133361659, pp: 569.

Kanvel, T.N. and E.C. Monie, 2009. Performance measure of different wavelets for a shuffled image compression scheme. IJCSNS Int. J. Comput. Sci. Netw. Securty, 9: 215-221.
Lillesand, T.M., R.W. Kiefer and J.W. Chipman, 2008. Remote Sensing and Image Interpretation. 6th Edn., John Wiley and Sons, Hoboken, ISBN: 9780470052457, pp: 756.

Mallat, S.G., 1989. A theory for multiresolution signal decomposition: The wavelet representation. IEEE Trans. Patt. Anal. Mach. Intell., 11: 674-693. DOI: 10.1109/34.192463

Sadashivappa, G. and K.V.S.A. Babu, 2008. Performance analysis of image coding using wavelets. IJCSNS Int. J. Comput. Sci. Netw. Secu., 8: 144-151. 